\title{
Antifungal Activity of Fluconazole, Itraconazole, Voriconazole, Amphotericin B and Caspofungin against Candida parapsilosis Blood Isolates
}

\author{
Stephanie Villalobos-Castro ${ }^{1}$, Daniela Jaikel-Víquez ${ }^{1,2 *}$, Diego \\ Ortiz-Solano $^{1}$, Luis Enrique Chaves-González ${ }^{1}$ and Norma T Gross ${ }^{1,2}$ \\ 1Section of Medical Mycology, Department of Microbiology and Immunology, \\ School of Microbiology, University of Costa Rica, San Pedro, Costa Rica \\ 2Tropical Disease Research Center (CIET), School of Microbiology, University of \\ Costa Rica, San Pedro, Costa Rica \\ *Corresponding Author: Daniela Jaikel-Víquez, Section of Medical Mycology, \\ Department of Microbiology and Immunology, School of Microbiology, University of \\ Costa Rica, San Pedro, Costa Rica.
}

Received: June 19, 2020

Published: August 26, 2020

(C) All rights are reserved by Daniela Jaikel-

Víquez., et al.

\begin{abstract}
Candida albicans is considered the most frequent etiological agent of candidemia worldwide. However, in the last decades there has been a rise in non-albicans Candida spp. causing candidemia. For example, in some Latin American countries Candida parapsilosis is replacing $C$. albicans as the main species isolated from blood samples. There is also an emerging concern regarding the decrease in susceptibility of non-albicans Candida spp. to first-line antifungals. In Costa Rica, there is limited information about this emerging problem. Thus, the objective of the present investigation was to study the susceptibility pattern of $C$. parapsilosis blood isolates to fluconazole, itraconazole, voriconazole, caspofungin and amphotericin B. Sixty-nine isolates collected from three Type A hospitals were studied. The isolates are part of the Fungal collection of the School of Microbiology, University of Costa Rica. Two reference methods were used to determine antifungal susceptibility. The EUCAST was performed for the azoles and amphotericin B. The end-points for caspofungin are not established by the EUCAST, thus the CLSI method was used instead. Results showed that $29 \%$ of the isolates were resistant to fluconazole, $25 \%$ to voriconazole, $6 \%$ to itraconazole and $3 \%$ to caspofungin. As to amphotericin no resistance was found; however, it is noteworthy that $42 \%$ of the isolates had minimal inhibitory concentrations of $1 \mu \mathrm{g} \mathrm{ml}{ }^{-1}$, the upper limit to be considered as sensible. In conclusion, these results highlight the importance of vigilance programs for susceptibility testing of $C$. parapsilosis involved in candidemia in Costa Rica.
\end{abstract}

Keywords: Amphotericin B; Azoles; Candida parapsilosis; Candidemia; Caspofungin; Fluconazole; Itraconazole; Susceptibility Testing; Voriconazole

\section{Abbreviations}

CLSI: Clinical Laboratory and Standards Institute; EUCAST: European Committee for Antimicrobial Susceptibility Testing; MIC: Minimal Inhibitory Concentration; MIC $_{50}$ : Minimal Inhibitory Concentration, which inhibits $50 \%$ of the isolates; $\mathrm{MIC}_{90}$ : Minimal Inhibitory Concentration, which inhibits $90 \%$ of the isolates; MFC: Minimal Fungicidal Concentration.

\section{Introduction}

Candida parapsilosis is an opportunistic yeast that causes both superficial infections in fingernails and feet, skin, middle ear and systemic infections; mainly in low weight premature neonates, catheter patients or receiving parenteral hyperfeeding [1]. This yeast is associated with the hands of the health area staff, including Costa Rica [2], which contributes to its nosocomial dissemination. Candida albicans is reported worldwide for approximately half of the cases of candidemia, followed by $C$. parapsilosis [1]. Even, in 
some Latin American countries, C. parapsilosis has displaced C. albicans as the main isolated species from blood cultures [3-5]. This is of clinical importance since species $C$. no-albicans such as $C$. parapsilosis, C. tropicalis, C. glabrata and C. krusei; are generally less susceptible to antifungals than C. albicans, implying different therapeutic management for this group of patients [1]. Clinical management of invasive candidosis with $C$. parapsilosis includes catheter removal and administration of systemic antifungals, such as amphotericin B, 5-fluorocyteine, echinocanine and some azoles such as fluconazole, itraconazole and voriconazole [6]. Amphotericin B is the most commonly used systemic antifungal; however, its use can cause nephrotoxicity, which forces to lower the doses or stop therapy $[7,8]$. The lipid formulation of amphotericin B is better tolerated [9-11] but for its high cost is not available in many countries such as Costa Rica. Therefore, although (2 - 3)\% in vitro resistance of $C$. parapsilosis to this antifungal [12] has been reported the biggest problem of the use of amphotericin B therapy is mainly its toxicity [13]. Fluconazole is the most common alternative treatment for amphotericin B. In vitro resistance to fluconazole has been demonstrated among the species of Candida no-albicans, particularly $C$. glabrata and $C$. krusei, the latter being intrinsically resistant to this medicine [14-16]. In addition, clinical resistance has been reported in C. parapsilosis in the case of this antifungal $[17,18]$. As for itraconazole, in vitro resistance of $1.5 \%$ [19] to 4\% [12] in isolations of C. parapsilosis has been observed. Voriconazole is effective for invasive disease and its clinical use has mainly been in oral therapies for patients with $C$. krusei and C. glabrata fluconazole-resistant and voriconazole-susceptible infection. Fluconazole and itraconazole have been shown to induce cross-resistance in C. parapsilosis and mutations have been identified in fluconazole and voriconazole resistant strains [20]. Due to this pattern, it is of relevance to carry out further studies on the in vitro sensitivity of these yeasts against the most widely used antifungals and thus to be able to guide the treating physician on what would be a better treatment scheme for patients with systemic thrush, decisive decision in the outcome of the clinical picture.

\section{Materials and Methods}

\section{Candida parapsilosis isolations}

The isolates studied are part of the collection of the Micoteca of the School of Microbiology of the University of Costa Rica. Sixtynine blood culture isolates were collected during the period of time between 1995 and 2018. The fungi were kept in Sabouraud glucose agar tubes at room temperature $((20-30) \mathrm{C})$. Control strains of the American Type Culture Collection: Candida krusei ATCC 6258 and Candida parapsilosis ATCC 22019 were used as controls for the susceptibility studies.

\section{Susceptibility tests}

TThe cutting points set by this method for amphotericin B are sensitive $\leq 1 \mu \mathrm{g} \mathrm{ml}^{-1}$ and resistant $>1 \mu \mathrm{g} \mathrm{ml}^{-1}$; for fluconazole, sensitive $\leq 2 \mu \mathrm{g} \mathrm{ml}^{-1}$ and resistant $>4 \mu \mathrm{g} \mathrm{ml}^{-1}$; for itraconazole, sensitive $\leq 0,125 \mu \mathrm{g} \mathrm{ml}^{-1}$ and resistant $>0,125 \mu \mathrm{g} \mathrm{ml}^{-1}$ and for voriconazole, sensitive $\leq 0,125 \mathrm{~g} \mathrm{ml}^{-1}$ and resistant $>0,25 \mu \mathrm{g} \mathrm{ml}^{-1}$. Because the cut-off points for caspofungin are not established by the EUCAST, the Clinical Laboratory and Standards Institute (CLSI) M27-A3 Broth Microdilution Method Standards (CLSI) [22] was used. The cutting points by this method are: sensitive $\leq 2 \mu \mathrm{g} \mathrm{ml}^{-1}$ and resistant $>2 \mu \mathrm{g} \mathrm{ml}^{-1}$.

\section{Statistical analysis}

Each trial was conducted in triplicate. For statistical analysis, the SPSS program for Windows, version 20 (SPSS Inc., Chicago, Ill, USA) was used. An average of the minimal inhibitory concentratio (MIC) was obtained with its respective standard error and the $\mathrm{MIC}_{50}$ and $\mathrm{MIC}_{90}$ for each antifungal. A variance analysis (ANOVA) was then performed to determine whether there are statistically significant differences between the values of MICs between antifungals and between hospitals, along with Tukey testing.

\section{Results and Discussion}

C. parapsilosis is a major cause of fungemia worldwide ((20 $30) \%$ ), the incidence of which has increased dramatically over the past two decades [4]. C. parapsilosis is found within the species of the genus Candida that are increasing as causal agents of candidemias in Australia, Latin America, Canada, Asia, Africa and Europe [23]. It is one of the most common species causing candidemia in hospitalized patients. In Europe; for example, it is the second or third most common agent by country, being responsible for (15 23)\% of all candidomic episodes. On the other hand, in the United States, $C$. parapsilosis is the third most common agent in patients over 12 years $(12 \%)$ and the second most common in younger patients (34\%). In Brazil and other Latin American countries, $C$. parapsilosis is responsible for (20 - 30)\% of candidemias, showing similar prevalence in adult and paediatric populations [24]. In some cases, $C$. parapsilosis has surpassed $C$. albicans as a causal species of candidemia, with a mortality rate ranging from 4 to $45 \%$, with an average of $28.5 \%$ [23].

Despite the growing relevance of candidemias, to date there are only three publications in Costa Rica in this regard, two from Hospital San Juan de Dios and one from Hospital México. In the first study [25], the clinical records of 47 adult patients, treated at the Hospital San Juan de Dios and diagnosed with systemic thrush be- 
tween 1996 and 1998, were analyzed. All had some risk factor for candidemia (previous use of antibiotics, central venous catheter, among others). C. albicans was reported to be the most common species (47\%), followed by C. tropicalis (19\%) and C. parapsilosis (11\%). In the second study conducted, five years later, $C$. parapsilosis ranked first [26]. In the latest study, between 2007 and 2010 [5] Candida species other than C. albicans are reported to have accounted for $62 \%$ of the causes of candidemia at Hospital México, which coincides with what is reported in other centers in Latin America and the world [5]. However, although the percentage of Candida no albicans so high coincides with what is reported for the region (collectively the non-albican species found outweigh $C$. albicans), the difference was found that $C$. parapsilosis was the most common cause of candidemia, displacing $C$. albicans as the most common species of all and its predominance in 3 of the 4 years analyzed show it as a problem endemic in that hospital. This differs from what is described in most reports, including Latin America, where $C$. albicans is the most common species (although in total percentage of candidemias it is exceeded by the sum of the percentage of non-albican species) and C. parapsilosis occupies the second or third place, with an incidence ranging from $10 \%$ to $25 \%$ [5]. In addition, in recent decades multiple studies have reported the emergence and increase of resistance to the most widely used ampfungals, where case C. parapsilosis [1] is of importance.

This study analysed 69 isolations from blood cultures in three hospital centers in the metropolitan area. $65 \%(n=45)$ of the isolates came from hospital A, 32\% $(n=22)$ from hospital B and 3\% $(n=2)$ from hospital C. When analysing the isolates of the three hospitals as a whole, $29 \%(\mathrm{n}=20)$ of resistance to fluconazole was found, $25 \%(n=17)$ to voriconazole, $6 \%$ (n s 4 ) to itraconazole and $3 \%(\mathrm{n}=2)$ to caspofungin. It is important to note that statistically significant differences were found between the geometric means of the MICs of the antifungals ( $\mathrm{F}=19,992 ; \mathrm{df}=4 ; \mathrm{p}<0.001)$. The Tukey test distributed the treatments in two groups, in the first were itraconazole, voriconazole, caspofungin and amphotericin B, which had the lowest MICs and in the second included fluconazole, with significantly higher MICs. Table 1 presents the distribution of geometric means, the range and the Inhibitory Minimum Concentrations (MICs) of the isolates.

In hospital A two fluconazole-resistant isolates were found, two to itraconazole, two to voriconazole and two to caspofungin (4.44\% resistance for each of the four antifungals) and one of the isolates was resistant to both fluconazole and voriconazole, but not

\begin{tabular}{|c|c|c|c|c|c|}
\hline & $\begin{array}{c}\text { Fluconazole } \\
\left(\mu \mathrm{gL}^{-1}\right)\end{array}$ & $\begin{array}{c}\text { Itraconazole } \\
\left(\mu \mathrm{gL} \mathrm{mL}^{-1}\right)\end{array}$ & $\begin{array}{l}\text { Voriconazol } \\
\left(\mu \mathrm{gL}^{-1}\right)\end{array}$ & $\begin{array}{l}\text { Amphotericin } \\
\left(\mu \mathrm{gL} \mathrm{m}^{-1}\right)\end{array}$ & $\begin{array}{l}\text { Caspofungina } \\
\left(\mu \mathrm{mL}^{-1}\right)\end{array}$ \\
\hline Geometric mean & $9,56( \pm 16,76)$ & $0,09( \pm 0,07)$ & $0,25( \pm 0,43)$ & $0,66( \pm 0,31)$ & $1,54( \pm 0,70)$ \\
\hline Range & $0,25-64,00$ & $0,03-0,50$ & $0,03-2,00$ & $0,19-1,00$ & $0,25-4,00$ \\
\hline $\mathrm{MIC}_{50}$ & 1,50 & 0,06 & 0,03 & 0,50 & 2,00 \\
\hline $\mathrm{MIC}_{90}$ & 32,00 & 0,13 & 1,00 & 1,00 & 2,00 \\
\hline
\end{tabular}

Table 1: Distribution of the minimum inhibitory concentrations (MICs) of C. parapsilosis isolations from three public Class A hospitals in Costa Rica, obtained from blood cultures, against fluconazole, itraconazole, voriconazole, amphotericin B and caspofungin ( $\mathrm{n}=69)$.

to itraconazole. In addition, itraconazole-resistant insulation was observed, but sensitive to fluconazole and voriconazole. The two caspofungin-resistant insulations were shown to be sensitive to the three azoles studied. In hospital B, 82\% ( $n=18)$ of resistance to fluconazole was observed, $68 \%(n=15)$ to voriconazole and $9 \%(n$ $=2$ ) to itraconazole and all were sensitive to caspofungin. The 15 voriconazole-resistant isolates were also resistant to fluconazole. The two isolates from hospital $\mathrm{C}$ were sensitive to the antifungals studied. It should be noted that statistically significant differences were found between fluconazole and voriconazole MICs between hospitals ( $\mathrm{F}=15,590 ; \mathrm{df}=2 ; \mathrm{p}<0.001)$ and $(\mathrm{F}=12,042 ; \mathrm{df}=2 ; \mathrm{p}<$ $0.001)$, respectively). In both cases, tukey-tested grouped hospitals $\mathrm{A}$ and $\mathrm{C}$ into one group and $\mathrm{B}$ in the other.
Se has reported Decreased sensitivity has been reported to $C$. parapsilosis to first-line antifungals, such as fluconazole [27]. Different studies have correlated the presence of resistance to fluconazole and of the pathogen isolation site, where yeasts obtained from the bloodstream are those that have such resistance. Interestingly, prolonged use of fluconazole to control C. parapsilosis candidemia in Finland led to the emergence of a resistant strain responsible for cross-infections for a period of 12 years [17]. However, the prophylaxis of this antifungal has not caused resistant strains in studies conducted over a period of 14 to 30 months [28,29]. As mentioned above, significant resistance to voriconazole (25\%) was also observed and to a lesser extent itraconazole (6\%). Although not common, resistance to itraconazole and voriconazole have also been reported [30]. 
In this study, 3\% resistance to caspofungin was observed. Compared to other species, C. parapsilosis tends to have high CMIs for echinocandines, which can lead to greater use of fluconazole for treatment and thus lead to resistance to azol. Also, it raises concern resistance to equinocandins, so that none of the first-line treatments [31] can be counted. Currently, the cause of decreased sensitivity to equinocandins is under study, but it has been seen that this species has polymorphisms occurring naturally in the FKS gene, which leads to changes in the corresponding subunit of glucan-synthetase and, therefore, a reduced action of the antifungal. In the case of amphotericin B, both increased MICs and resistance have been reported [31].

All the isolates in the present study were sensitive to amphotericin. Amphotericin B resistance is not common, so amphotericin B MICs for C. parapsilosis are reported at average MIC values MIC $_{50}$ and $\mathrm{MIC}_{90}$ between $\left(0.13\right.$ - 1) $\mu \mathrm{g} \mathrm{ml}^{-1}$ and $(0.5-1) \mu \mathrm{g} \mathrm{ml}^{-1}$ respectively [16,32-34]. In our study, it was observed that $42 \%(n=29)$ of the isolates analysed had MICs equal to $1 \mathrm{~g} \mathrm{ml}^{-1}$ for amphotericin $\mathrm{B}$, the upper limit to be classified as sensitive. Initially, it could be argued that strains are developing resistance mechanisms that allow them to survive in increasing concentrations of amphotericin B. However, we cannot suggest a decrease in the sensitivity of amphotericin B because tolerance to this antifungal by this species has been described. The reason for such behaviour has not been clarified; however, it was described since 1983, where a comparative study was conducted between seven different species of Candida with different antifungals. In this work it was observed that the minimum fungicide concentration (MFC) for C. parapsilosis isolations was up to 32 or more times its corresponding MICs, while the other species had MFCs less than 16 times their corresponding CMIs, so only C. parapsilosis met the definition of tolerance to amphotericin $B$ (the same criterion was used as defining tolerance for bacterial agents). The author proposes that this tolerance may be due to the composition of cell membrane sterols of this species, very similar to that observed in other Amphotericin resistant Candida spp. [35]. The same characteristic was observed in 2003, except that Candida dubliniensis was also listed as tolerant. In another study conducted in 1998, it was observed that in cultures of density between $(0.5$ - 1.0) x $10^{6}$ cells $\mathrm{ml}^{-1}$ supplemented with $2 \mu \mathrm{g} \mathrm{ml}^{-1}$ amphotericin B, C. parapsilosis together with C. krusei were the species that had the lowest susceptibility to the antifungal, with a decrease of viable cells of only 1 to 2 magnitudes. C. parapsilosis was the least sensitive to amphotericin B of all, with a survival rate of $1 \%$ even after 24-hour exposure [36]. Another example of this behavior was reported in 2004, in a study comparing the patterns of the rate of elimination of amphotericin B over different Candida species. Unlike the other species where the fungicide point was reached between 2 to 4 hours of incubation with 4 times the antifungal MIC, a 48-hour incubation was required to reach it with $C$. parapsilosis MIC [37]. One study reported that C. parapsilosis is the species with the highest percentage of hydrophobicity in its cell wall compared to the other species analyzed and the second with the most biofilm formation capacity (only after $C$. albicans). The authors argue that these two characteristics play an important role in resistance to antifungals, disinfectants and even factors in the host [38]. This could help to clarify the mechanisms of tolerance to amphotericin B of $C$. parapsilosis; however, more studies are needed in order to determine the reason for that characteristic.

A link has been established between proper hand washing and decreased incidence of candidemia for $C$. parapsilosis. Since this species is in the hands of humans, iti is important to diminish the amout of yeast in the hands of health personnel [5,39] . A proper decrease in the amount of yeast through this practice would lead to less exposure of this species to antifungals administered to patients, reducing the chances of resistance development and the selection of already resistant strains themselves. Another factor to consider is a good protocol and guidelines for the administration of antifungals established for the treatment of candidemias. In Costa Rica, there is no protocol given by the Ministry of Health or the Costa Rican Social Security Fund, considering the results of the present study", it is recommended to carry out tests of sensitivity to first-line antifungals in the candidemia caused by $C$. parapsilosis to provide adequate treatment and thus reduce mortality in patients, prolonged hospital stay and increase in care costs.

\section{Conclusion}

In the present study, the data obtained from a collection of 69 C. parapsilosis bloodstream isolates from three public hospitals in Costa Rica demonstrated that $29 \%$ were resistant to fluconazole, $25 \%$ to voriconazole, $6 \%$ to itraconazole and $3 \%$ to caspofungin. These results emphasize the need for surveillance programs to include first line antifungal profiles of $C$. parapsilosis involved in candidemia in Costa Rica.

\section{Acknowledgements}

We would like to thank the University of Costa Rica for financial support (Projects VI-803-B9-024 and VI-430-B7-732) and Alejandra Gómez Arrieta, Assistant of the Medical Mycology Laboratory, School of Microbiology, University of Costa Rica for her valuable support.

\section{Conflict of Interest}

The authors have no conflicting interests to declare. 


\section{Bibliography}

1. Trofa D., et al. "Candida parapsilosis, an emerging fungal pathogen”. Clinical Microbiological Reviews 21 (2008): 606-625.

2. Carrillo-Dover P., et al. "Isolation of Candida spp. and other yeasts in the staff who work in critical areas of the Hospital San Juan de Dios". Costa Rican Medical Act 51 (2009): 166-171.

3. Nucci M., et al. "Epidemiology of opportunistic fungal infections in Latin America". Clinical Infectious Diseases 51.5 (2010): 561-570.

4. Tapia C., et al. "Candida parapsilosis complex". Chilean Journal of Infectology 32 (2015): 569-570.

5. Villalobos J., et al. "Epidemiology of candidemia at Hospital México". Costa Rican Medical Act 58 (2016): 15-21.

6. Barchiesi F., et al. "Caspofungin in combination with amphotericin B against Candida parapsilosis". Antimicrobial Agents of Chemotherapy 51 (2007): 941-945.

7. Walsh TJ., et al. "Echinocandins-an advance in the primary treatment of invasive candidiasis". New England Journal of Medicine 347 (2002): 2070-2072.

8. Moudgal VT., et al. "Multiechinocandin- and multiazole-resistant Candida parapsilosis isolates serially obtained during therapy for prosthetic valve endocarditis". Antimicrobial Agents of Chemothererapy 49 (2005): 767-769.

9. Oppenheim B A., et al. "The safety and efficacy of amphotericin B colloidal dispersion in the treatment of invasive mycoses". Clinical Infectious Diseases 21 (1995): 1145-1153.

10. Adler-Shohet F., et al. "Amphotericin B lipid complex for neonatal invasive candidiasis". Archives of Disease in Childhood Fetal and Neonatal Edition 84 (2001): F131-F133.

11. Linder N., et al. "Treatment of candidaemia in premature infants: comparison of three amphotericin B preparations". Journal of Antimicrobial Chemotherapy 52 (2003): 663-667.

12. Ostrosky-Zeichner L., et al. "Antifungal susceptibility survey of 2,000 bloodstream Candida isolates in the United States". Antimicrobial Agents of Chemotherapy 47 (2003): 3149-3154.

13. Ellis D. "Amphotericin B: spectrum and resistance". Journal of Antimicrobial. Chemotherapy 49.1 (2002): 7-10.

14. Pfaller MA., et al. "International surveillance of bloodstream infections due to Candida species: frequency of occurrence and antifungal susceptibilities of isolates collected in 1997 in the United States, Canada, and South America for the SENTRY Program". Journal of Clinical Microbiology 36 (1998): 18861889.
15. Tortorano AM., et al. "The European Confederation of Medical Mycology (ECMM) survey of candidaemia in Italy: antifungal susceptibility patterns of 261 non-albicans Candida isolates from blood". Journal of Antimicrobial Chemotherapy 52 (2003): 679-682.

16. Fleck R., et al. "In vitro susceptibility of Candida species to five antifungal agents in a German university hospital assessed by the reference broth microdilution method and E test". Journal of Antimicrobial Chemotherapy 59 (2007): 767-771.

17. Sarvikivi E., et al. "Emergence of fluconazole resistance in a Candida parapsilosis strain that caused infections in a neonatal intensive care unit". Journal of Clinical Microbiology 43 (2005): 2729-2735.

18. Tumbarello M., et al. "Biofilm production by Candida species and inadequate antifungal therapy as predictors of mortality for patients with candidemia". Journal of Clinical Microbiology 45 (2007): 1843-1850.

19. Arias, A., et al. "In vitro susceptibility of 545 isolates of Candida spp. to four antifungal agents". Mycoses 37 (1994): 285-289.

20. Branco J., et al. "Fluconazole and voriconazole resistance in Candida parapsilosis is conferred by gain-of-function mutations in MRR1 Transcription Factor Gene". Antimicrobial Agents of Chemotherapy 59 (2015): 6629-6633.

21. Subcommittee on Antifungal Susceptibility Testing (AFST) of the ESCMID European Committee for Antimicrobial Susceptibility Testing (EUCAST). "EUCAST Definitive document Edef 7.1: method for the determination of broth dilution MICs of antifungal agents for fermentative yeasts". Clinical Microbiology and Infection 14 (2008): 398-405.

22. Clinical and Laboratory Standards Institute. "Reference method for broth dilution antifungal susceptibility testing of yeasts, approved standard. CLSI Document M27-A3". Clinical and Laboratory Standards Institute, Wayne, PA. (2008).

23. Moreno X., et al. "Frequency and in vitro sensitivity profile of Candida parapsylosis complex isolations from patients with candidemias". Journal of the Venezuelan Society of Microbiology 35 (2015): 103-110.

24. Gonçalves S., et al. "Prevalence rates and antifungal susceptibility profiles of the Candida parapsilosis species complex: results from a nationwide surveillance of candidaemia in Brazil". Clinical Microbiology and Infection 16 (2010): 885-887.

25. Caballero E., et al. "Systemic Candidiasis-Experience at the Hospital San Juan de Dios 1996-1998". Costa Rican Journal of Medical Sciences 20 (1999): 153-165. 
26. Mora N., et al. "Candidemia at San Juan de Dios hospital from 2003 to 2005". College of Microbiologists and Clinical Chemists 11 (2005): 17-19.

27. Wanjare S., et al. "Candida parapsilosis: an emerging pathogen, Its distribution and antifungal susceptibility to fluconazole and amphotericin B". International Journal of Health Sciences and Research 7.4 (2017): 119-126.

28. Kaufman D., et al. "Fluconazole prophylaxis against fungal colonization and infection in preterm infants". New England Journal of Medicine 345 (2001): 1660-1666.

29. Kicklighter SD., et al. "Fluconazole for prophylaxis against candidal rectal colonization in the very low birth weight infant". Pediatrics 107 (2001): 293-298.

30. van Asbeck EC., et al., "Candida parapsilosis: a review of its epidemiology, pathogenesis, clinical aspects, typing and antimicrobial susceptibility". Critical Reviews in Microbiology 35.4 (2009): 283-309.

31. Silva AP., et al. "Prevalence, distribution, and antifungal susceptibility profiles of Candida parapsilosis, C. orthopsilosis, and C. metapsilosis in a tertiary care hospital". Journal of Clinical Microbiology 47.8 (2009): 2392-2397.

32. Kuhn DM., et al. 2002. "Antifungal susceptibility of Candida biofilms: unique efficacy of amphotericin B lipid formulations and echinocandins". Antimicrobial Agents of Chemotherapy 46 (2002): 1773-1780.

33. Marco F., et al. "Trends in frequency and in vitro susceptibilities to antifungal agents, including voriconazole and anidulafungin, of Candida bloodstream isolates. Results from a sixyear study (1996-2001)". Diagnostic Microbiology Infectious Diseases. 46 (2003): 259-264.

34. Merkerová M., et al. "Cloning and characterization of Sapp2p, the second aspartic proteinase isoenzyme from Candida parapsilosis". FEMS Yeast Research 6 (2006): 1018-1026.

35. Seinfield SM., et al. "Amphotericin B tolerance: a characteristic of Candida parapsilosis not shared by other Candida species". Journal of Infectious Diseases 147.1 (1983): 116-119.

36. Vazquez JA., et al. "Stable phenotypic resistance of Candida species to amphotericin B conferred by Preexposure to subinhibitory levels of azoles. Journal of Clinical Microbiology 36.9 (1998): 2690-2695.

37. Cantón, E., et al. "Patterns of amphotericin B killing kinetics against seven Candida species. Antimicrobial agents and Chemotherapy" 48.7 (2004): 2477-2482.
38. Dabiri S., et al. "Comparative analysis of proteinase, phospholipase, hydrophobicity and biofilm forming ability in Candida species isolated from clinical specimens". Journal de Mycologie Medicale 28.3 (2018): 437-442.

39. Bonassoli L A., et al. "High frequency of Candida parapsilosis on the hands of healthy hosts". Journal of Hospital Infection 59.2 (2005): 159-162.

\section{Assets from publication with us}

- Prompt Acknowledgement after receiving the article

- Thorough Double blinded peer review

- Rapid Publication

- Issue of Publication Certificate

- High visibility of your Published work

Website: www.actascientific.com/

Submit Article: www.actascientific.com/submission.php

Email us: editor@actascientific.com

Contact us: +919182824667 